\title{
The complete mitogenome of Fusarium culmorum
}

\author{
Tomasz Kulik , Balazs Brankovics², Jakub Sawicki ${ }^{1,3}$, and Anne van Diepeningen ${ }^{2}$ \\ ${ }^{1}$ Department of Botany and Nature Protection, University of Warmia and Mazury in Olsztyn, Olsztyn, Poland, ${ }^{2}$ CBS-KNAW Fungal Biodiversity \\ Centre, Utrecht, the Netherlands, and ${ }^{3}$ Department of Biology and Ecology, University of Ostrava, Ostrava, Czech Republic
}

\begin{abstract}
The structure of the Fusarium culmorum mitogenome is similar to that of closely related Fusarium spp.: it has a total length of $103,844 \mathrm{bp}$, the base composition of the genome is the following: A (35.4\%), T (32.9\%), C (14.6\%), and G (17.1\%). The mitogenome contains 13 proteincoding genes, 2 ribosomal RNA (rRNA), and 28 transfer RNA (tRNA) genes, all coded on the same strand of DNA. The gene order is identical to that of the other Fusarium and Hypocreales mitogenomes. Maximum likelihood and Bayesian analysis based on the concatenated amino acid dataset of mitochondrial protein-coding genes confirmed close genetic relationship of F. culmorum to the other type B trichothecene producers F. graminearum and F. gerlachii.
\end{abstract}

\section{Keywords}

Fungi, Fusarium culmorum, mitogenome

\section{History}

Received 5 March 2015

Revised 9 March 2015

Accepted 14 March 2015

Published online 27 May 2015
Fusarium culmorum is an ubiquitous soil-borne fungus able to cause foot and root rot and Fusarium head blight on different small-grain cereals. It causes yield and quality losses and results in contamination of the grain with mycotoxins (deoxynivalenol, nivalenol, and zearalenone), which are a potential health hazard for both humans and animals (Scherm et al., 2013). We sequenced the complete mitogenome (mitochondrial genome) of the F. culmorum strain CBS 139512 (GenBank accession number KP827647) isolated from wheat head in North Poland (Tywęzy village) in 2003 and deposited in the CBS Fungal Biodiversity Centre, Utrecht, The Netherlands. Whole genome libraries were prepared using a Nextera XT kit (Illumina Inc., San Diego, CA) from genomic DNA extracted from mycelium. Constructed libraries were sequenced on the Illumina Miseq platform (Illumina Inc., San Diego, CA) with the 250 bp pairedend read, version 2. De-multiplexed and trimmed reads were aligned to the reference sequence of the $F$. graminearum $\mathrm{PH}-1$ strain (Cuomo et al., 2007). Revised PH-1 mitogenome is available in NCBI under accession number HG970331. Sequence reads were aligned with Geneious (v.6.1.6 created by Biomatters, South Plainfield, NJ, available from http://www.geneious.com). The initial mitogenome annotation was carried out using MFannot (Mega Sun Inc., Saint Louis, MO) (http:// megasun.bch.umontreal.ca/cgi-bin/mfannot/mfannotInterface.pl) and manually adjusted. Annotation of tRNA genes was improved using tRNAscan-SE (Barry Stoddard, Seattle, WA) (Pavesi et al., 1994). Annotation of protein coding genes was corrected by aligning intronless homologs to the genome. The position of the intron in the $r n l$ gene was identified by mapping RNA-Seq reads
(SRP039087) to the mitogenome of PH-1 strain, and aligning the resulting intron to the current genome. Intron-encoded proteins were identified using ORF Finder (Bioinformatics, Walnut Creek, CA) (http://www.ncbi.nlm.nih.gov/gorf/gorf.html) and annotated using InterPro (InterPro Inc., Lisle, IL) (http:// www.ebi.ac.uk/interpro/). The $F$. culmorum mitogenome structure is similar to the closely related $F$. graminearum $\mathrm{PH}-1$ mitogenome (Cuomo et al., 2007); it has a total length of $103,844 \mathrm{bp}$, the base composition of the genome is the following: A (35.4\%), T (32.9\%), C (14.6\%), and $\mathrm{G}(17.1 \%)$, the $\mathrm{G}+\mathrm{C}$ content $(31.7 \%)$ is similar to that of the other Fusarium mitogenomes. The mitogenome of $F$. culmorum is a circular DNA molecule containing two ribosomal RNAs, one ribosomal protein gene rps3, 13 protein-coding genes. and 28 tRNA genes. The tRNA genes range in size from $71 \mathrm{bp}$ to $88 \mathrm{bp}$, and code for all 20 standard amino acids. The gene order is identical to the one of the PH-1 strain, as well as to other Fusarium mitogenomes and all are coded on one of the DNA strands. Comparison of $F$. culmorum and $F$. graminearum mitogenomes showed an $8206 \mathrm{bp}$ size difference, which was primarily due to the presence of three-group I introns in F. culmorum: cox 2 intron 3 , cob intron 1, and cox 3 intron 3 . Using phylogenetic analysis (Figure 1), F. culmorum, $F$. graminearum, and $F$. gerlachii formed well-supported clade (1/100) that is in accordance with previous studies showing close genetic relationship of $F$. culmorum to the other type B trichothecene producers (Kristensen et al., 2005). This new mitochondrial genome will provide vital clues on the evolutionary history of F. culmorum. 
Figure 1. Phylogenetic tree of the mitochondria of Fusarium culmorum and related species based on the concatenated amino acid dataset of mitochondrial protein-coding genes. The concatenated amino acid dataset of mitochondrial protein-coding genes of F. culmorum (KP827647), F. graminearum (HG970331), F. gerlachii, (KM486533), F. oxysporum (NC017930), F. verticillioides (NC016687), F. solani (NC016680), F. fujikuroi (JX910420), F. circinatum (JX910419), and Verticillium dahliae (DQ351941) were aligned using Clustal W implemented in Geneious software (Biomatters, South Plainfield, NJ) with the default settings. Maximum Likelihood analysis was conducted using Mega 6 (MEGA Inc.,

Englewood, NJ) (Tamura et al., 2013) where the stability of clades was assessed by 1000 bootstrap replications, while MrBayes 3.1 (Applied Biosystems Inc. (ABI), Foster City, CA) was used for Bayesian inference using Markov chain Monte Carlo (MCMC) methods to estimate the posterior distribution of model parameters (Ronquist et al., 2012).

Values at branches indicate branch support

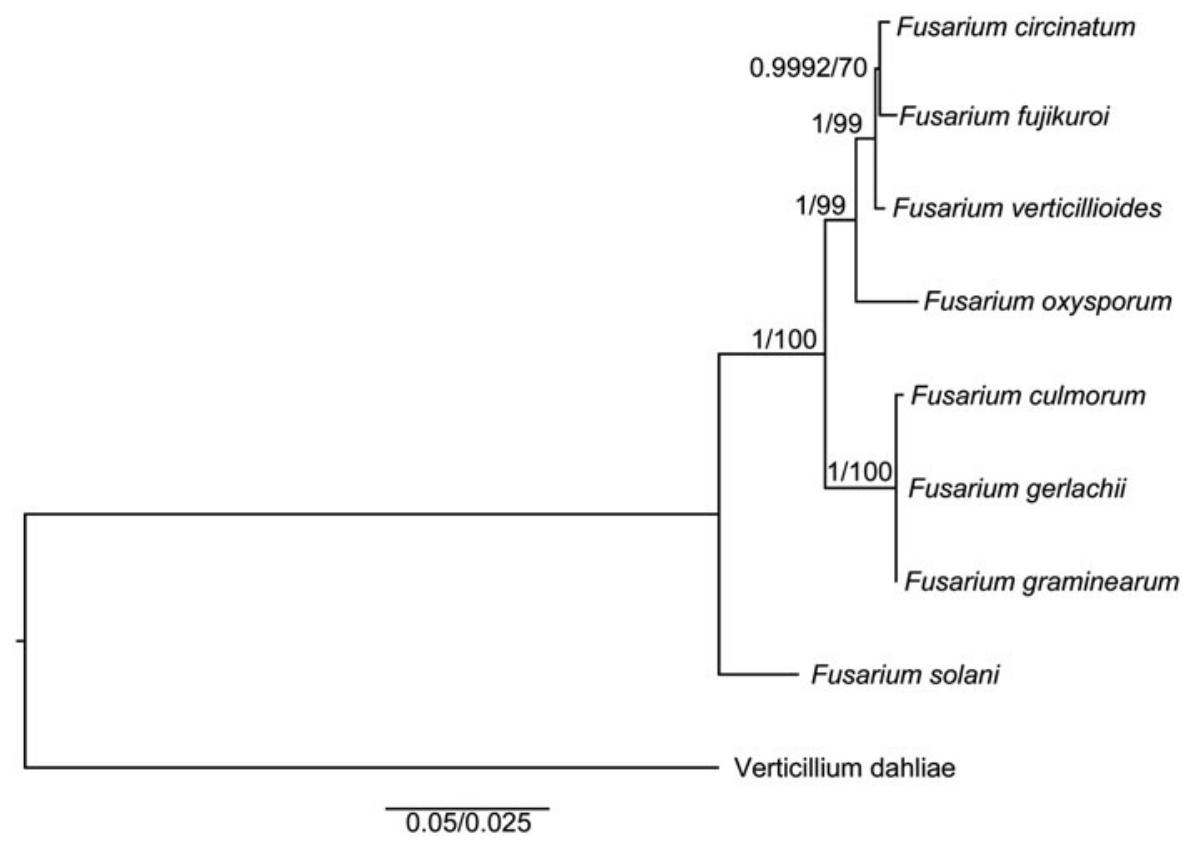
posterior probabilities inferred with Bayesian analysis/bootstrapping percentages based on the maximum likelihood analysis.

\section{Declaration of interest}

The authors report that they have no conflicts of interest. The authors alone are responsible for the content and writing of the paper. This work was funded by the Grant DEC-2013/11/B/NZ9/01788 from the National Science Center, Poland.

\section{References}

Cuomo CA, Güldener U, Xu JR, Trail F, Turgeon BG, Di Pietro A, Walton JD, et al. (2007). The Fusarium graminearum genome reveals a link between localized polymorphism and pathogen specialization. Science 317:1400-2.

Kristensen R, Torp M, Kosiak B, Holst-Jensen A. (2005). Phylogeny and toxigenic potential is correlated in Fusarium species as revealed by

partial translation elongation factor 1 alpha gene sequences. Mycol Res 109:173-86.

Pavesi A, Conterio F, Bolchi A, Dieci G, Ottonello S. (1994). Identification of new eukaryotic tRNA genes in genomic DNA databases by a multistep weight matrix analysis of transcriptional control regions. Nucl Acids Res 22:1247-56.

Ronquist F, Teslenko M, van der Mark P, Ayres DL, Darling A, Höhna S, Larget B, et al. (2012). MrBayes 3.2: Efficient Bayesian phylogenetic inference and model choice across a large model space. Syst Biol 61: 539-42.

Scherm B, Balmas V, Spanu F, Pani G, Delogu G, Pasquali M, Migheli Q. (2013). Fusarium culmorum: Causal agent of foot and root rot and head blight on wheat. Mol Plant Pathol 14:323-41.

Tamura K, Stecher G, Peterson D, Filipski A, Kumar S. (2013). MEGA6: Molecular evolutionary genetics analysis version 6.0. Mol Biol Evol 30:2725-9. 